\title{
PARAMETERS THAT INFLUENCE THE ULTRASONIC BOND QUALITY
}

\author{
P.L.L.M. DERKS \\ Centre for Technology, N.B. Philips' Gloeilampenfabrieken, Eindhoven, Netherlands \\ (Received June 5, 1981; in final form June 26, 1982)
}

\begin{abstract}
This paper summarizes some non-destructive ultrasonic bond quality test methods. Systems described in the literature are mainly based on monitoring the deviation of the vibration amplitude during welding and the correlation between the bond strength with these deviations. Practical application of these principles can give discouraging results. A better understanding of the bond formation process should enable one to understand what causes the amplitude to deviate, and why this is strongly dependent on the ultrasonic bond system involved.
\end{abstract}

\section{INTRODUCTION}

In the micro-electronics industry there is a tendency to introduce fully automated, highspeed ultrasonic or thermosonic wire bonders. It would be of great value if reliable and fast non-destructive bond quality test methods could be incorporated. The desired system should detect any weak bond, without damaging good bonds, and should operate on any type of wire bonder, independent of wire or product properties. In addition, the system could be of use in setting up optimal bond parameters.

Non-destructive pull-test methods ${ }^{1}$ answer these requirements to a certain extent. Unfortunately the reliability is strongly influenced by geometrical variables and wire properties. Establishing adequate pull force levels for each bond remains the main problem. Acoustic-emission techniques have been suggested as of future importance in reliability improvement. ${ }^{2}$ However, no applications in wire bonding have been reported as yet. The most promising methods are based on the observation that the vibration amplitude behaviour of the bonding tool can sometimes be correlated successfully to the bond quality. Microphone measurements of tool amplitudes near the vibration node ${ }^{3}$ did not give good correlation between the signals measured and bond quality. An inprocess quality control system which has already been tested in production, measures amplitudes directly, and is called: "Amplitude Deviation Detector" (ADD)., ${ }^{4,5}$ Other sources $^{6,9}$ also indicate that it is indeed possible to design monitoring systems that approximate the requirements mentioned above.

Application of the ADD principle in various production situations has sometimes given discouraging results. Therefore some work has been carried out to study the bond parameters and properties of the ultrasonic welding apparatus in relation to bond quality and amplitude deviation. The results are presented here.

\section{BOND PARAMETERS}

Parameters that influence the bond quality can be divided into two groups: machine parameters and system parameters. There are four independent machine parameters: clamping force $(\mathrm{F})$, amplitude of tool vibration $(\mu)$, bond time $(\mathrm{t})$ and temperature. Under ideal conditions one can find optimum combinations of these parameters, which will lead to good bonds. The system parameters are, however, of equal importance for good bond- 
ability. They are: type of ultrasonic resonator, tool material and geometry, stiffness of product clamping, kind and condition of metallisation, wire properties, etc.

Production disturbances, resulting in weak bonds, can be caused either by variations of machine parameters or by inproper system parameters. Of course, many parameters cannot be monitored, so a practical in-process bond quality monitoring system will measure parameters related to the performed bond itself (e.g. wire deformation, bond strength).

\section{THE ADD SYSTEM}

The vibration amplitude of an unloaded tool is proportional to the power setting of the ultrasonic generator. During bond formation, however, the tool is submitted to mechanical forces. In general this causes the amplitude to change. This amplitude can be measured, for instance, at the end face of the transducer with an optical displacement sensor (Fotonic Sensor), which gives a signal directly proportional to the tool amplitude. ${ }^{4,5}$ When the transducer is fed from a constant voltage source, the transducer current is proportional to its mechanical vibration amplitude. Application of this property facilitates an in-process amplitude measurement.

During bonding, characteristic deviations from the freely vibrating amplitude can occur (Figure 1a). Under certain circumstances this deviation can be correlated to the bond quality. For weak bonds, e.g. when welding onto contaminated substrates, bond curves as shown in Figure 1b may be obtained. The ADD system ${ }^{4,5}$ measures the freely vibrating amplitude and compares it with the amplitude after a certain pre-set delay time during the welding pulse. The difference is monitored as a percentage of the freely vibrating amplitude. For production application the limiting deviation has to be determined, and a direct correlation between amplitude deviation and bond quality must be found. In order to be able to adapt the ADD principle to different bonding equipment and to interpret the deviation curves, it is necessary to know in more detail what causes the amplitude deviation.

\section{WIRE DEFORMATION AND AMPLITUDE DEVIATION}

All bonding experiments were carried out with a standard $60 \mathrm{kHz}$ piezoelectric ultrasonic bonder with a tungsten carbide tool. The power was supplied from a constant voltage
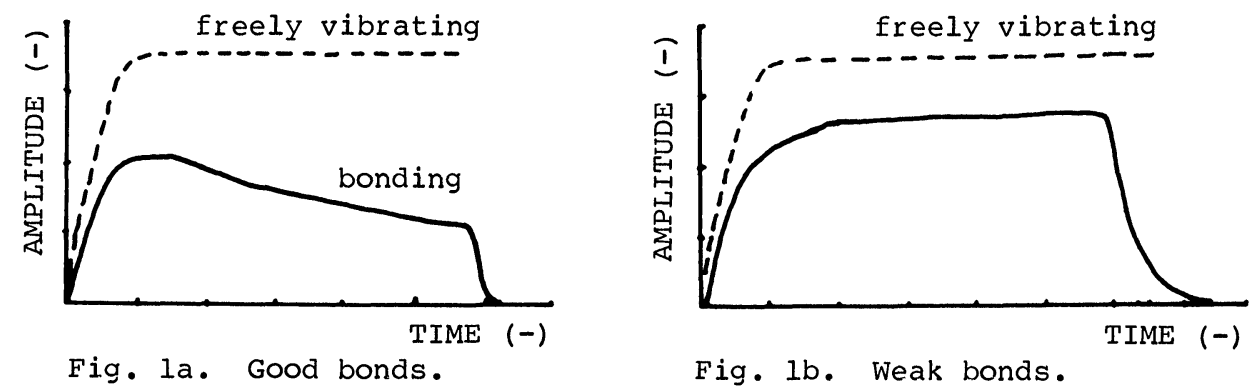

FIGURE 1 Amplitude deviation during bonding (arbitrary units). 
generator, the output impedance was matched to the bonding process. Aluminium $1 \% \mathrm{Si}$ wire of $30 \mu \mathrm{m}$ diameter (elongation $1 \%$, tensile strength $0.22 \mathrm{~N}$ ) was bonded onto gold plated $(3 \mu \mathrm{m})$ ceramic. Only wedge type bonds were investigated. The bond strength was determined by a $30^{\circ}$ angle pull test (Figure 2 ).

According to Harman ${ }^{1}$ and Florian, ${ }^{7}$ the bond strength is correlated with the plastic wire deformation. The deformation is defined here as the vertical tool displacement during the welding pulse (see Figure 2). The relationship between strength and deformation in our experiments is shown in Figure 2. Below $11 \mu \mathrm{m}$ deformation the bonds are too weak and will give failure in the wire-substrate interface (lift-off). The optimal deformation in our case is found to be 14-18 $\mu \mathrm{m}$. Too small or excessive deformation gives low bond strength. Practical experience confirms that weak bonds, e.g. due to improper system parameters, very often correlate with incorrect wire deformations. Unfortunately, in-process measurement of wire deformation (a few microns) can be troublesome in production lines. Knowing that the amplitude deviation can sometimes be correlated to bond strength and that bond strength is correlated to wire deformation, we investigated the amplitude deviation and wire deformation. Aluminium wires were bonded onto clean substrates. In our experiments the vibration amplitude and wire deformation were measured during bonding (Figure 3). Both power setting (level of freely vibrating amplitude) and clamping force were varied. The transducer vibration amplitude was determined by measuring the transducer current. The wire deformation was measured with an optical non-contacting displacement sensor (Fotonic Sensor). Both signals were displayed on an oscilloscope. The curves obtained proved to be very reproducible.

The freely vibrating amplitude increases exponentially to a constant level. The rise time depends on generator and resonator properties, and can be as large as $20 \mathrm{~ms}$. This fact has to be taken into account when determining the amplitude deviation during the whole bond time. After an initial increase, the amplitude during bonding decreases to a constant value which is determined by the freely vibrating amplitude level and clamping force. The main wire deformation is reached in a time which is short with respect to normal bond times (i.e. about $30 \mathrm{~ms}$ ). The deformation depends on clamping force and amplitude.

\section{DISCUSSION}

From curves like those in Figure 3 it was considered if there is a relationship between amplitude deviation and deformation. The amplitude deviation is the difference between

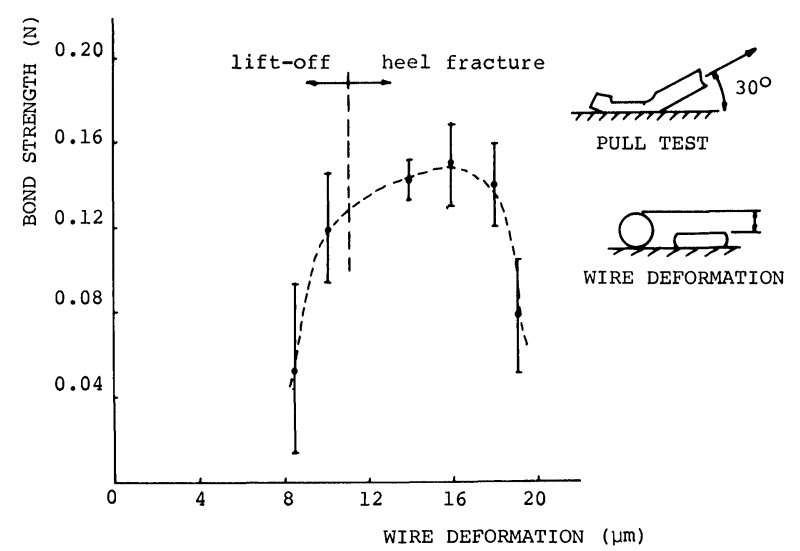

FIGURE 2 Bond strength versus wire deformation (30 $\mu \mathrm{m} \mathrm{Al} \mathrm{wire).}$ 
freely vibrating and bond amplitude. Figure 4 shows an almost linear relationship, which only depends on the clamping force. From this we conclude that the deformation, at any power setting and at any time, can be controlled by measuring the amplitude deviations.

In order to deform the wire, a certain amount of plastic deformation energy is required, which at constant bond time will be proportional to the power setting on the generator. Figure 5 shows that this assumption holds. From Figures 4 and 5 it can be concluded that for a certain bond time and clamping force, the amplitude deviation must be proportional to the freely vibrating amplitude and independent of the deformation. From this it follows that the relative amplitude deviation (the amplitude deviation divided by the freely vibrating one) will be constant for a given bond time and clamping force. For
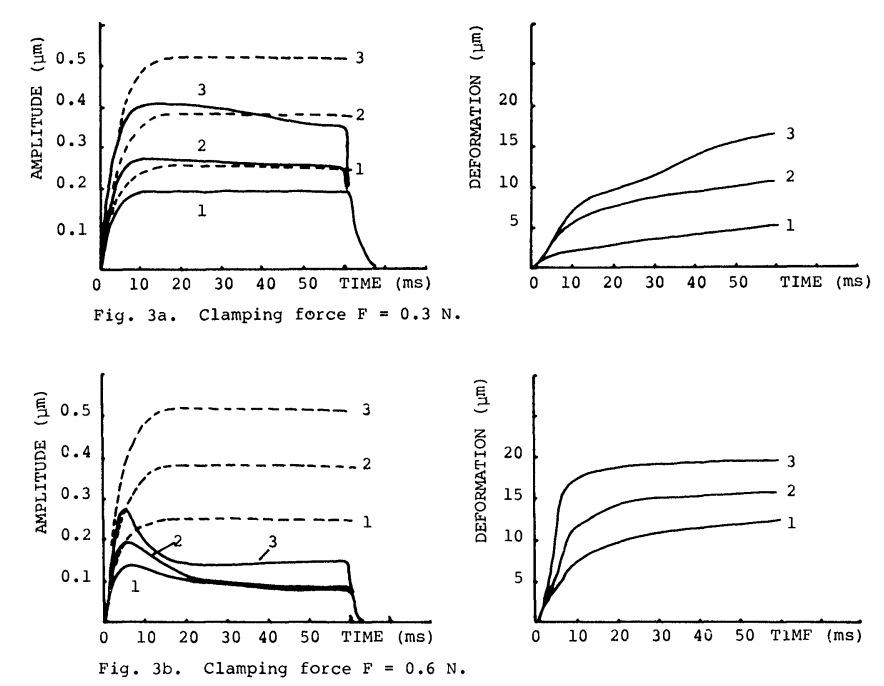

FIGURE 3 Vibrational amplitude and wire deformation versus time; freely vibrating amplitudes $(---): 1=0.25 \mu \mathrm{m}, 2=0.38 \mu \mathrm{m}, 3=0.52 \mu \mathrm{m}$.

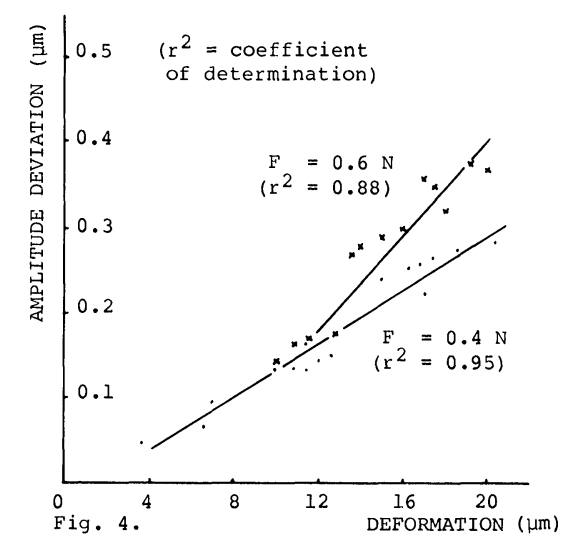

FIGURE 4 Amplitude deviation versus wire deformation during bond formation; clamping force $\mathrm{F}=0.4$ and $0.6 \mathrm{~N}$. 


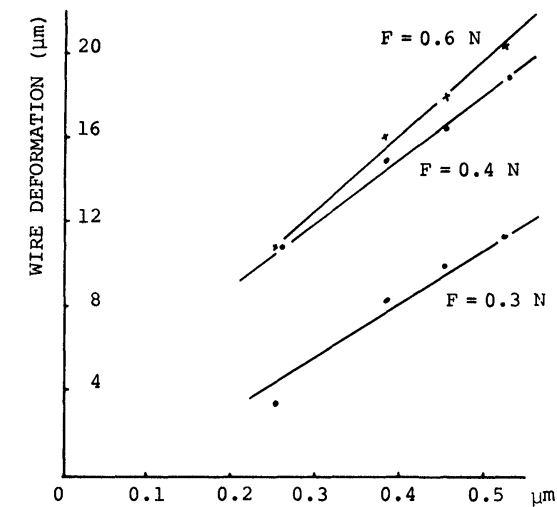

FIGURE 5 Wire deformation versus freely vibrating amplitude; bond time $t=30 \mathrm{~ms}$, clamping force $\mathrm{F}=0.3,0.4$ and $0.6 \mathrm{~N}$.

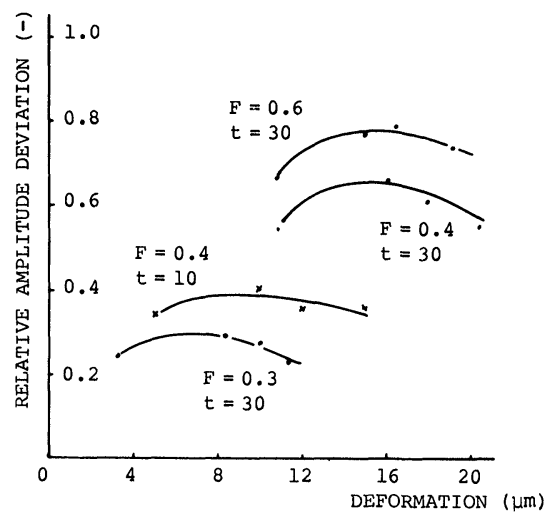

FIGURE 6 Relative amplitude deviation versus wire deformation with the power setting as a parameter. Various combinations of clamping force and bond time $(\mathrm{F}=0.3,0.4$ and $0.6 \mathrm{~N}, \mathrm{t}=10$ and $30 \mathrm{~ms})$.

some combinations of clamping force and bond time the relative amplitude deviation and wire deformation were measured at different power settings (Figure 6). Indeed, the relative amplitude deviation hardly depends on wire deformation.

In practice the bond parameters are determined by variation of the power setting, at a pre-set bond time and clamping force (say $t=30 \mathrm{~ms}$ and $\mathrm{F}=0.4 \mathrm{~N}$ ), until an acceptable bond strength (= deformation) is reached. From Figure 5 we learn that in this way the relative amplitude deviation will give no information at all on the wire deformation or bond quality. Within the acceptable deformation range $(12-18 \mu \mathrm{m})$ (see also Figure 2) where strong bonds are made, the relative amplitude deviation is practically constant. But also outside this range with a low bond strength, it has the same value.

Further, it is found that for a certain wire deformation, say $14 \mu \mathrm{m}$, and so a certain bond strength, the relative amplitude deviation strongly depends on bond time and clamping force. So, even if the relative amplitude deviation may not be of help in setting bond parameters, it is quite useful in monitoring machine parameter deviations. A reduction in bond time or clamping force changes the relative amplitude deviation level. From Figure 4 it follows that the amplitude deviation itself will give more information on the wire deformation or bond strength. Further experiments were carried out to support this. 
TABLE I

Bond strength at constant wire deformation (clamping force $\mathrm{F}=0.4 \mathrm{~N}$; mean values of 10 measurements).

\begin{tabular}{llllll}
\hline $\begin{array}{l}\text { deformation } \\
(\mu \mathrm{m})\end{array}$ & $\begin{array}{l}\text { freely vibrating } \\
\text { amplitude }(\mu \mathrm{m})\end{array}$ & $\begin{array}{l}\text { bond time } \\
(\mathrm{ms})\end{array}$ & $\begin{array}{l}\text { bond strength } \\
(\mathrm{N})\end{array}$ & $\begin{array}{l}\text { amplitude } \\
\text { deviation }(\mu \mathrm{m})\end{array}$ & $\begin{array}{l}\text { relative } \\
\text { deviation }(-)\end{array}$ \\
\hline $16 \pm 1$ & 0.33 & 100 & $0.19 \pm 0.01$ & $0.23 \pm 0.01$ & 0.70 \\
$16 \pm 1$ & 0.36 & 30 & $0.18 \pm 0.02$ & $0.23 \pm 0.01$ & 0.64 \\
$16 \pm 1$ & 0.55 & 12 & $0.17 \pm 0.01$ & $0.22 \pm 0.01$ & 0.40 \\
\hline
\end{tabular}

A constant bond strength will be obtained, when the wire deformation is kept constant. Table I reveals that for different combinations of power settinv and bond time an almost constant strength can be obtained. The amplitude deviation is constant indeed, whereas the relative deviation is not.

From the experiments we conclude that the deviation magnitude of vibration amplitude of the welding tool is correlated to the wire deformation. The in-process monitoring of the amplitude deviation makes it possible to control the bond quality. When amplitude deviation is kept constant, the wire deformation and so the bond strength will be constant too.

\section{SYSTEM PARAMETERS}

The influence of tool length and tool material has been reported. ${ }^{4,5}$ Depending on the tool length we found amplitude deviation curves completely different from those in Figure 3. For a certain tool length one will measure amplitude deviation curves which are the same for good bonds as for weak bonds. No discrimination between bonding on clean or on contaminated substrates will be found, which seems to make an amplitude deviation system useless. Therefore, before designing a quality monitoring system, tool amplitude transformation curves have to be determined, in order to be able to choose a tool length that will give sufficient amplitude deviation, which gives information on the wire deformation. A tool length which gives low amplitude transformation (see Figure 7) in general will give little amplitude deviation sensitivity for various wire deformations. A tool with high amplitude transformation (near tool resonance) will be sensitive to load variations. Depending on the length chosen, the amplitude will decrease or increase during bonding. The tool and resonator transform mechanical load impedances into electrical impedances at the electrical terminals of the transducer. Variation of the generator output impedance with respect to the transducer input impedance will also influence the system sensitivity. This was confirmed by experiments. So, the applicability of a bond quality monitoring system based on amplitude deviations can strongly be influenced by the system parameters.

\section{CONCLUSIONS}

1) Amplitude deviation during ultrasonic wire bonding can be correlated to the wire deformation.

2) Determination of amplitude deviation levels for bond quality acceptance necessitates a thorough parameter study for each application.

3) The deviation magnitude is strongly influenced by system parameters like tool length and impedance matching. 


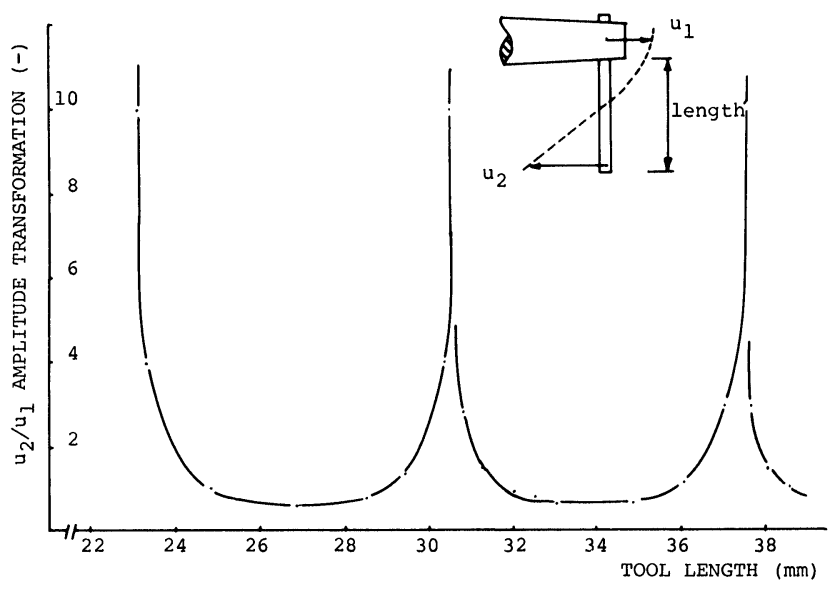

FIGURE 7 Measurements of amplitude transformation for a freely vibrating tool $(60 \mathrm{kHz})$, diameter $=1.6 \mathrm{~mm}$, tool steel).

\section{ACKNOWLEDGEMENTS}

The author wishes to thank Mr. J. de Vries for his assistance in carrying out the experiments, and Dr. G. Tichelaar for his valuable advice in preparing the manuscript.

\section{REFERENCES}

1. G.G. Harman, "A metallurgical basis for the non-destructive wire-bond pull-test". 12th Annual Proceedings IEEE Reliability Physics, pp. 205-210, (April 2-4, 1974).

2. G.G. Harman, "The use of acoustic emission in a test for beam lead bond integrity". 14th Annual Proceedings IEEE Reliability Physics, pp. 86-97, (April 20-22, 1976).

3. J.H. Albers, "In process bond monitor". NBS Special Publication 400-29, (1976).

4. R.V. Winkle, "A new method of quality control for ultrasonic wire bonding". Conference Proceedings, Ultrasonics International 1979, pp. 62-68, 15-17 May, Graz, Austria.

5. M. Hight, R. Winkle, J. Dale, Improvements in and relating to ultrasonic bonding apparatus. British Patent No. 1506164.

6. A.I. Belyakow, "Automatic monitoring of the ultrasonic welding process". Svar. Proiz., No. 7, pp. 45-47, (1976).

7. W. Florian, "Miktro-Ultraschallschweissverbindungen von Golddrähten auf Halbleiterbaustein" (in German). Industrie-Elektronik, 20. Jahrgang Nr. 17/18 pp. 341-343, (1979).

8. G.J. Mulder, private information, rep. 1977.

9. V.A. Rodionov, "The problems of stabilizing the quality of microwelded joints". Presented at IIW Annual Assembly, Lissabon, Portugal, Doc. IV-283-80, (1980). 

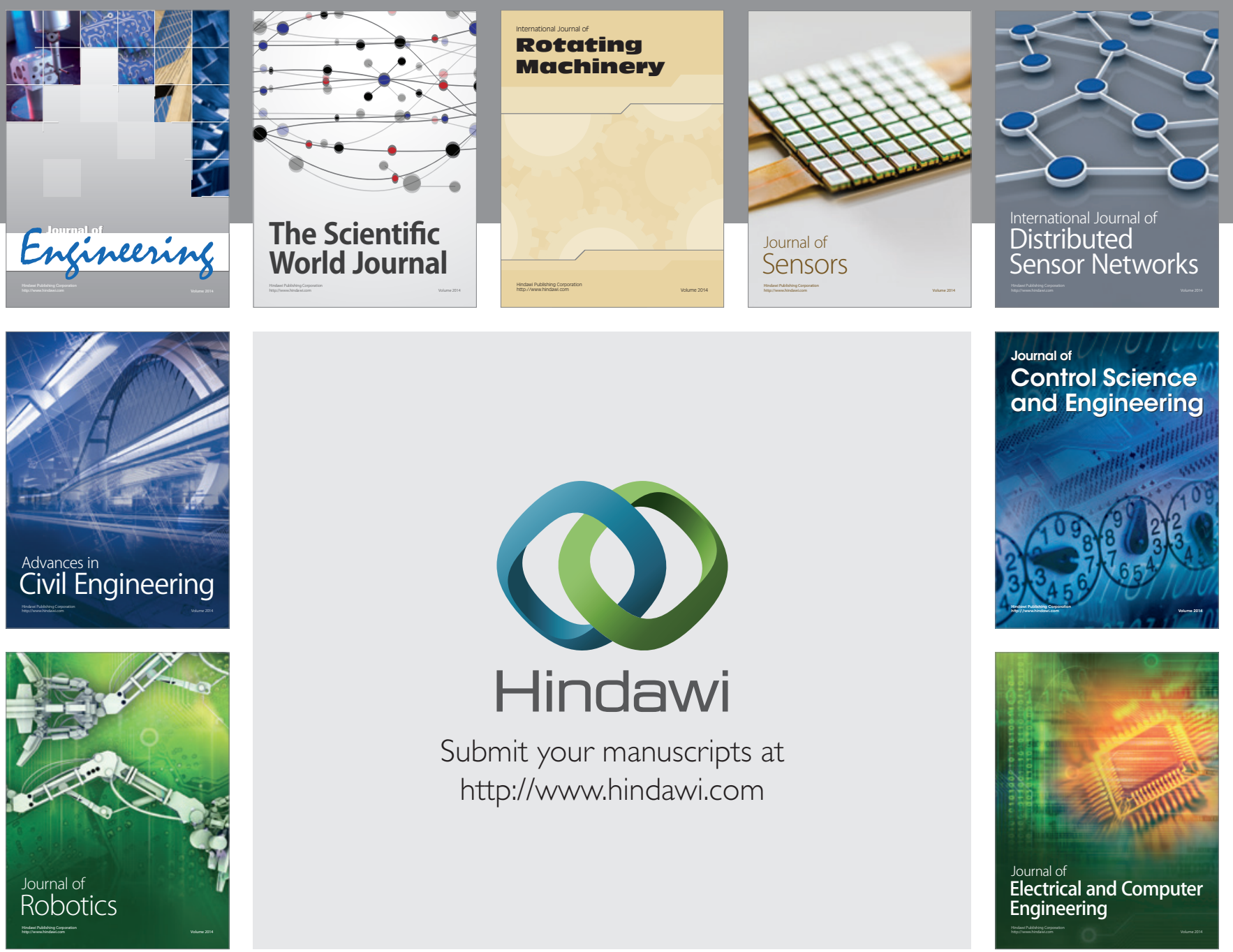

Submit your manuscripts at

http://www.hindawi.com
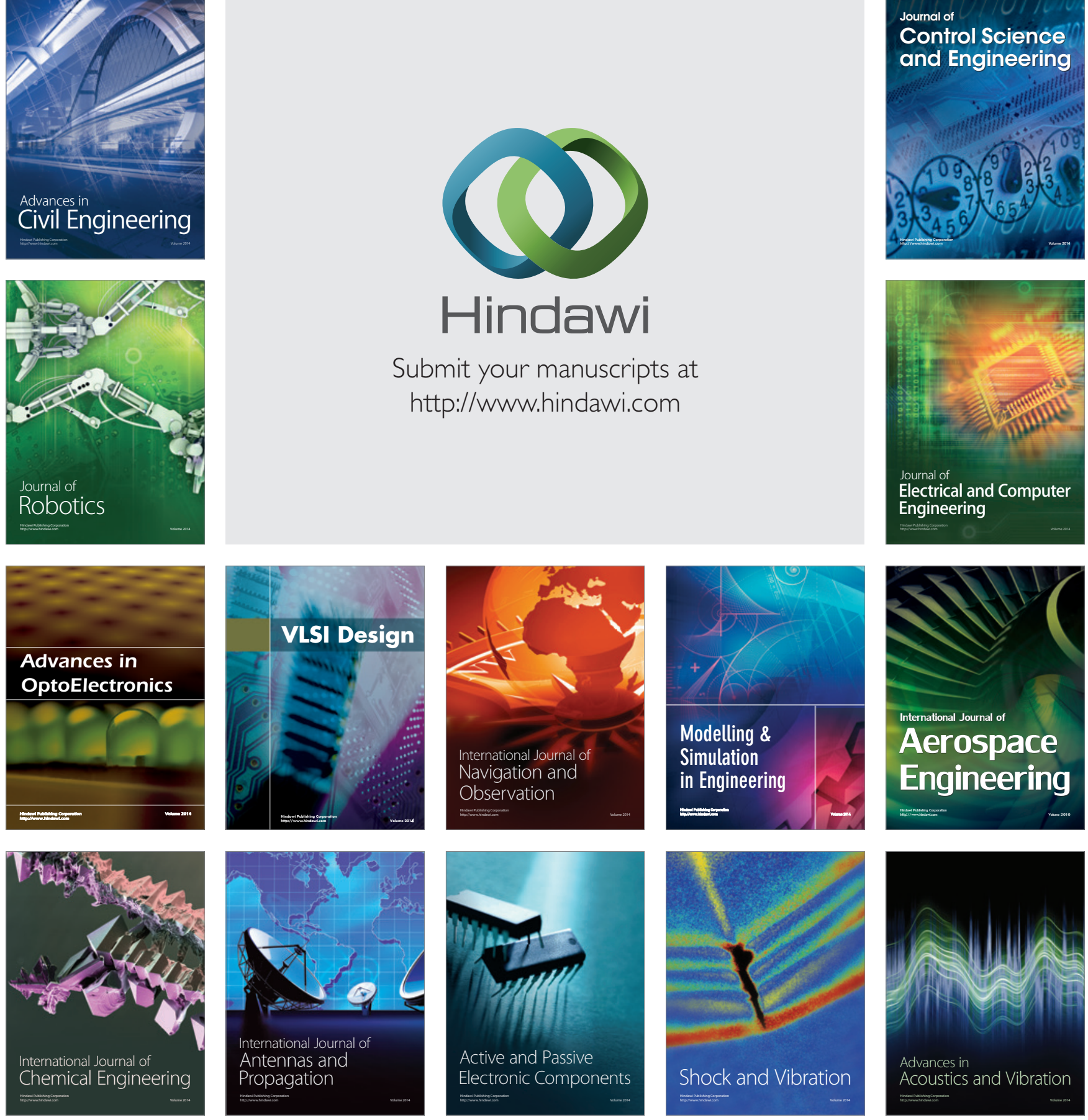